B e ckm an $\mathbf{n s}$ Zeiten her an den Universitäten geblüht hatte, an die neugegründeten technischen Hochschulen verwiesen wurde, war man wohl der Ansicht, daß auch eine entsprechende Scheidung der Studentenschaft erfolgen werde. Tatsächlich ist dies aber nicht der Fall gewesen, da ja auch die überwiegende Zahl der an den Universitäten studierenden Chemiker später in die Industrio ïbertritt. Aus verschiedenen, insbesondere materiellen Gründen besteht oft nicht die Möglichkeit, die Lücken der Universitatsbildung durch weiteres Studium an der Hochschule auszufüllen. Unter den gegenwärtigen Umständen wírd die Freizugigkeit der Studenten in noch stärkerem Maße beeinträchtigt werden, d. h. es wird der Mehrzah] der Studierenden nur mehr das Studium an Heimatsorte möglieh sein. Und was anderen Fächern recht ist, miüBte der angewandten Chemie billig sein - niemand denkt etwa daran, die klinischen Semester der Studenten an Alrademien für praktische Medizin zu verweisen, obwohl z. B. geburtshelferische ṫbungen am Fantom sicher nicht nehr als "rein wissenschaftlich" bezeichnet werden können. Auch in Rechtswissenschaft und Theologie gibt es mancher. lei Lehrfächer, die vom Standpunkt des strengen Puristen ans nicht an die Universität gehören, wie überhaupt die Universitäten in viclen Fächern einen gewissen fachschulmäBigen Einschlag aufweisen, wenn man sich dessen auch meist nicht bewußt ist.

Wie nun das Fach der Technologie auszugestalten ist und durch was für Persönlichkeiten es zu besetzen ist, ergibt sich aus dem Vorhergesagten eigentlich schon von selbst. Unbedingte Grunderfordernis ist, daß $\beta$ die Technologie den Tecbnologen verbleibt; die anderen Forderungen nach Ordinariaten oder Extraordinariaten treten daneben zurück, wenn auch mindestens selbständige Institutsabteilungen wünschenswert wären. Jedenfalls wäre zu erstreben, daß nach Möglichkeit eine reinliche Scheidung zwischen dem Techno logen, dem Chemiker und dem Nahrungsmittelchemiker einträte. Die Technologie ist eine besondere Wissenschaft neben der Chemie erst wenn dieser Grundsatz sich Bahn gebrochen hat, wird eine Gesundung der Verhältnisse möglich sein. Die Definition, wer als Technologe anzusehen wäre, ist nicht ganz leicht. Es müßte entweder industrielle Praxis Vorbedingung sein oder tatsächlich ausschlieBliche oder vorwiegendo technologisehe (techniseh-chemische) Tätigkeit; diese Tätigkeit hätte entweder in praktischer Labora. toriumstätigkeit auf dem Gebiet der angewandten Chemie zu bestehen oder in theoretischer Forschungstätigkeit etwa auf technischwirtschaftlichem Gebiete. Unter allen Umständen aber müßten volkswirtschaftliche Ausbildung und Interessen gefordert werden. Bei ilteren Dozenten werden diese meist auf autodidaktischem Wege erworben worden sein; für den Nachwuchs aber müßte auch die Möglichkeit gesichert werden, Volkswirtschaftslehre als Examensnebenfach zu nehmen. Diese Ausbildungsmöglichkeiten verbessern nicht nur den Nachwuchs für die Praxis und die wissenschaftliche Technologie, sondern sie werden dem Chemiker auch den Aufstieg in die Verwaltungsämter erleichtern, in denen er, sicher oft zum Schaden der Allgemeinheit, selbst auf seinem eigentlichen Fachgebiet gegenüber den Juristen nicht diejenige Rolle spielt, die Volkswirtschaftler und Ingenieure auf ihrem entsprechenden Gebiete bereits $\mathrm{zu}$ erringen im Begriffe sind. Zurzeit jedenfalls ist sogar umgekehrt selbst in der Industrie gelegentlich ein Zurüekweichen des Chemikers vor dem Juristen zu beobachten, offenbar weil es an volkswirtschaftlich - und verwaltungstechnisch - geschulten Chemikern fehlt, so daß die Direktorenposten großer Werke in steigendem Maße mit Juristen besetzt werden.

Vielleicht werden diese Zeilen diesen oder jenen Kollegen zu Meinungsäußeruungen veranlassen und hoffentlich wird die Bewegung der Technologen trotz der materiell ungünstigen Umstände nicht wieder verebben, wie es bisher noch jedesmal der Fall war. Es ist ferner zu wünschen, daß auch das preußische Kultusministerium bei der Errichtung und Besetzung von Lehrstühlen und der Erteilung. von Lehraufträgen, soweit es die Mittel zulassen, künftig dem Charakter der Technologie als besonderer Wissensehaft Rechnung trùgt.

\section{Der Aufschluß von Phosphaten mit Bisulfat.}

Von Prof. Dr. BRrnhard Neumann u. Dipl.-Ing. Kort Kueylein. (Mitcellungen aus dem Instjtut für Chemische Tochnologie, Technische Hochsehule Breslau.)

(Eingeg. 5./1. 1920)

Auch in der Indistrie der Herstellung künstlicher Düngemittel hat der Krieg mancherlei schwierige Verhältnisse geschaffen. Die Einfuhr hochwertiger Rohphosphate ans dem Auslande stockte sehr bald und hörte schließlich ganz auf. Als Ersatz für diesen Ausfall mußten stark kreidehaltige deutsche, nordfranzösische und belgische Phosphate herangezogen werden, die schon wegen des starken Schwefelsäureverbrauchs ein ungünstiges Rohmaterial darstellen. Mindestens ebenso schwierig wurde die Beschaffung der großen Mengen dünner Schwefelsäure, welche die Superphosphatfabrikation braucht, da infolge der mangelnden Kieseinfuhr und anderweitigen Verbrauchs der Säure nicht mehr gentigende Mengen der Düngerindustrie zur Verfügung gestellt werden konnten. Um die zur Verfügung stehende Schwefelsäuremenge zu strecken, hatdann das Kriegs amt einen Ersatz der Säure durch Bisulfat; erst bis zu 1/6, dann bis zu 1/s ihres Gehaltes an Schwefelsäure vorgeschrieben. Die damit erzielten Aufschlußergebnisse waren jedoch in keiner Weise befriedigend, abgesehen von den sonstigen Mißständen, welche diese Ar beitsweise mit sich brachte.

Die wissenschaftliche Aufklärung der Mißerfolge dieses Schwefel säureersatzes durch Bisulfat fehlt bis jetzt, die nachstehenden Ausführungen und Untersuchungen werden die Begründung erbringen.

Es ist bekannt, daß man auch mit anderen Säuren als Schwefel säure Phosphate bis zu Superphosphat aufschließen kann. Beim AufschluB mit Salzsäure entsteht nebenher das hygroskopische und vielen Pflanzen schädliche Chlorcalcium, beim Aufschluß mit Salpetersäure Calciumnitrat, das zwar einen bestimmten Düngewert haben würde, aber ebenfalls zerfließlich ist, außerdem ist die Salpetersäure als Aufschlußmittel zu teuer. Schwefelsäure liefert dagegen neben dem wasserlöslichen Monocalciumphosphat das erforderliche Gipsskelett, welches in erster Linie die Streufähigkeit des Superphosphats bedingt.

Bisulfat ist nun auch schon früher, wemn auch in anderer Weise zum Aufschluß von Phosphaten vorgeschlagen worden. Jaehne1) hat schon 1891 auf die Verwendung von Bisulfat hingewiesen. Er benutzte eine Lösung von 1,5 Teilen Bisulfat mit 6 Teilen Wasser; er wollte die entstehenden löslichen Salze auslaugen, eindampfen und das erhaltene $\mathrm{Salz} 2 \mathrm{Na}_{2} \mathrm{SO}_{4} \cdot \mathrm{CaH}_{4}\left(\mathrm{PO}_{4}\right)_{2} \cdot \mathrm{H}_{2} \mathrm{O}$ verwenden. $\mathrm{Ma}$ t i g $n \circ n^{2}$ ) schlug dagegen vor, das heiße, bei der Salpetersäurefabrikation ablanfende Bisulfat in Wasser zu lösen und mit dieser sauren Lösung Rohphosphat aufzuschließen; das entstehende Superphosphat mußte aber dann noch getrocknet werden.

Wenn jetzt im Kriege Bisulfat als Ersatz eines Teiles der notwendigen Schwefelsäure Verwendung finden sollte, so gab es nur zwei Wege, um das durchzuführen, entweder mußte man das Rohphosphat mit Bisulfat vermahlen und das Gemisch dann im Rührkessel mit dem berechneten Minderbedarf an Schwefelsäure versetzen, oder man mußte die Schwefelsäure erst mit einem gewissen Prozentsatz Bisulfat zusammenbringen und diese dann auf das Rohphosphat einwirken lassen. Die erstere Methode gewährleistet entschieden die innigere Vermischung und vermeidet eine vorzeitige Klumpenbildung. Diese Methode ist allein in der Technik zur Anwendung gekommen.

Wenn das Bisulfat wirklich einen Teil der Schwefelsäure ersetzen kann, dann muß die im Bisulfat enthaltene freie Schwefelsäure bei der chemischen Umsetzung tatsächlich auch wirksam werden können. $\mathrm{Ob}$ das möglich ist, werden nachfolgende Betrachtungen zeigen.

Wir haben es hier mit einem System $\mathrm{NaHSO}_{4}-\mathrm{H}_{2} \mathrm{SO}_{4}$ zu tun. Bei den vorkommenden Konzentrationsverhältnissen dissoziieren $\mathrm{NaHSO}_{4}$ and $\mathrm{H}_{2} \mathrm{SO}_{4}$ in $\mathrm{Na}$ und $\mathrm{HSO}_{4}$, und $\mathrm{H}$ und $\mathrm{HSO}_{4}$. Nun ist allgemein bekannt, daß ionisierte Salze in Lösungen, die eines der Ionen des Salzes enthalten, schwerer löslich sind wie in reinem Wasser oder fremden Salzlösungen. Es gilt die Beziehung

$$
\frac{[\mathrm{Na}]\left[\mathrm{HSO}_{4}\right]}{\left[\mathrm{NaHSO} \mathrm{SO}_{4}\right]}=\text { konst. }
$$

Die Konzentration des nicht dissoziierten Teiles ist proportional dem Ionenprodukt. Frhöht man in einer Bisulfatlösung durch Zusatz von $\mathrm{H}_{2} \mathrm{SO}_{4}$ die Konzentration der $\mathrm{HSO}_{4}$-Ionen, so wäohst das Ionenprodukt und damit die Konzentration des undissoziierten Salzes abber das Lösungsgleichgewicht hinaus, und ein entsprechender Teil des undissoziierten Salzes muß als Bodenkörper ausfallen. Dadurch wird aber die Wirksamkeit der im Bisulfat vorhandenen freien Säure zurikckgedrängt. Je konzentrierter die verwendete Schwefelsäure ist, desto geringer muB der Dissoziationsgrad des Bisulfats sein. Hieraus folgt, daß bèim Ersatz eines Teiles der Schwefelsäure durch Bisulfat iberhaupt erst von einer bestimmten Säurekonzentration $a b$ b $\mathrm{r}$ t $\mathrm{s}$ von einem, Ersatz" die Rede sein kann. W e n $\mathbf{n}$

1) D. R. P. 57 295. Jahrb. d. Chem. Techn. 1891, 409.

2) Chem. Ind. 1893, 343; Chem.-Ztg. 1891, 1757. 
man also den Wert des Bisulfatzusatzes or. mitteln will, som a man in erster Liniefest. stellen, in welchen Grenzensich das Bisulfat in der zum AufschluB bestimmten Schwefels ä u r e löse $n$ l ä $\beta t$, denn das nicht gelöste Salz ist als toter Bodenkörper fü die Reaktion unwirksam.

Um den Grad der Iöslichkeit des Bisulfats in Schwefelsäure festzustellen, wurde wie folgt verfahren. In Schüttelgefäßen wurde ge nau titrierte Schwefelsäure verschiedener Konzentration mit einem Überschuß von Bisulfat versetzt, so daß stets ein Bodenkörper vorhanden war. Dieses Gemisch wurde auf einem Schüttelapparat mehrere Stunden lang geschüttelt. Dann wurde in eiriem bestimmten Volumen die Schwefelsäure mit $1 / 2-n$-Natronlauge von neuem titriert. Die dabei erhaltenen Ergebnisse der Versuchsreihen sind in nachstehender Tabelle zusammengestellt.

\begin{tabular}{|c|c|c|}
\hline $\begin{array}{c}\text { Grade } \\
\text { Bé. }\end{array}$ & $\begin{array}{c}\text { Durch Titration } \\
\text { ermittelte } \\
\text { g } \mathrm{H}_{2} \mathrm{SO}_{4} \text { in Liter }\end{array}$ & $\begin{array}{c}\text { Nach Sattigung mit } \\
\text { Bisulfat durch Titra- } \\
\text { tion erhalten } \\
\mathrm{g} \mathrm{H}_{2} \mathrm{SO}_{4} \text { in Liter }\end{array}$ \\
\hline 52,0 & 1021,0 & 997,0 \\
\hline 50,3 & 966,5 & 949,5 \\
\hline $46,4^{\prime \prime}$ & 844,9 & 851,0 \\
\hline 42,8 & 741,7 & 750,0 \\
\hline 38,6 & 632,2 & 650,5 \\
\hline 35,0 & 548,3 & 573,7 \\
\hline 31,3 & 468,1 & 522,7 \\
\hline 26,3 & 370,8 & 462,0 \\
\hline Gesätti & 廿äsśrige Bisu & jsung 344,0 . \\
\hline
\end{tabular}

Die Tabelle zeigt, daß nur bei niedrigen Säurekonzentrationen die Menge der Schwefelsäure nach der Sättigung mit Bisulfat zu genommen hat, daß dagegen bei hohen Säurekonzentrationen durch die Sättigung der Gehalt an Schwefelsäure sogar zurückgegangen ist. Es muB sich also auch irgendwo ein Punkt finden, wo ein wirklicher Gleiehgewichtszustand zwischen Salz und Säure vorhanden ist, wo also die konzentrierte Säure aus dem Salze so viel Wasser aufnimmt, daß dadurch gerade die Wirkung der freien Säure des Salzes kompensiert wird. Dieser Punkt liegt, wie sich aus der graphischen Darstellung entnehmen läßt, bei $47,5^{\circ}$ Bé. Diese Verhältnisse zeigt sehr deutlich das Kurvenblatt 1. Auf der Abezissenachse sind die

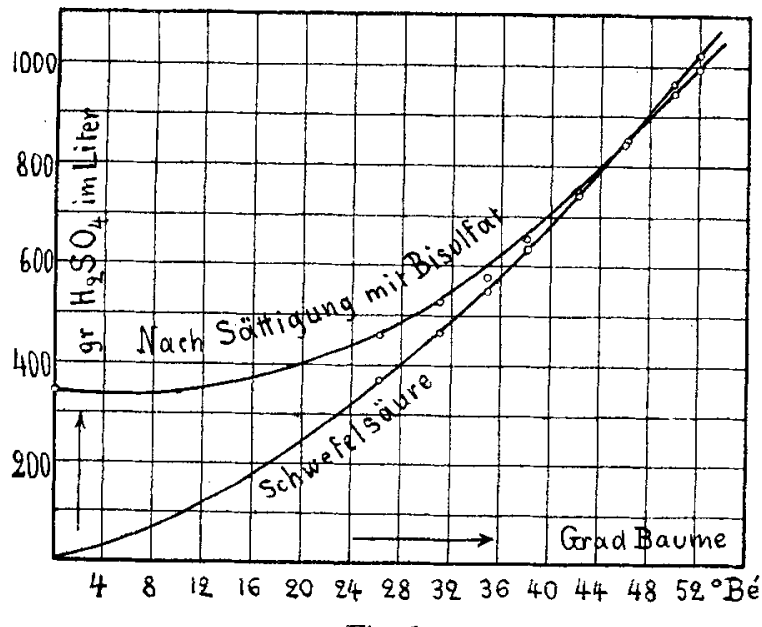

Fig. 1.

Säurekonzentrationen in Baumégraden eingetragen, auf der Ordinatenachse die Gramm Schwefelsäure im Liter. Die untere, im Schnittpunkte der beiden Achsen beginnende Kurve gibt die Gehalte der reinen Säure wieder, die zweite, anfangs höher liegende Kurve zeigt die Gehalte an Schwefelsäure der mit Bisulfat gesättigten Säure. Das. Bisulfat war ein technisches Bisulfat, welches 35,07\% freie, und $40,13 \%$ gebundene $\mathrm{H}_{2} \mathrm{SO}_{4}\left(=58,14 \% \quad \mathrm{Na}_{2} \mathrm{SO}_{4}\right)$ enthielt. Der Wassergehalt betrug demnach $6,79 \%$

Die Kurven zeigen nun, daß zu jeder Säurekonzentration eine bestimmte Löslichkeit des Salzes in der Säure gehört. Die Löslich keit des Bisulfats ist in dèr schwächsten Säure am größten und nimmt mit steigender Säurekonzentration ab, ganz wie es die Dissoziationsverhältnisse verlangen. Je höher die Säurekonzentration ist, desto mehr nimmt die Menge des Bodenkörpers, der an der Reaktion nicht teilnimmt, zll. Bei dem Schnittpunkt der beiden Kurven, welcher bei einer Säurekonzentration von $47,5^{\circ}$ Bé. liegt, ist also alles Bisulfat. als undissoziiertes Salz vorhanden. Oberhalb dieses Punktes geht durch den Bisulfatzusatz die Konzentration der Säure sogar zurück, weil die konzentrierte Säure dem nicht dissoziierten Salze das Krystallwasser entzieht und sich dadurch verdünnt.

Ein Ersatzder Schwefels äureduroh Bisulfat hat also eigentlich nur einen Sinn, wenn man mit niedrigen Säurekonzentrationen, etwa von $30^{\circ} \mathrm{Bé}$. an a b wärts arbeiten könnte. In der Praxis wäre also der Bisulfataufschluß erst mit solchen dünnen Säuren erfolgreich gewesen. Man schließt aber niemals praktisch mit derartig dünnen Säuren auf. Es hat auch keinen Zweck, die Kammersäure bis auf $30^{\circ}$ Bé. zu verdünnen, da selbst, wenn die Wirksamkeit der Säure durch Bisulfatzusatz etwas erhöht wird, das überschüssig eingeführte Wasser nachher wieder durch einen kostspieligen Darrprozeß ausgetrieben werden müßte.

Um die Richtigkeit der vorstehenden Ausführungen, daß nämlich letzten Endes die Löslichkeit des Bisulfats in den verschieden konzentrierten Säuren der maßgebende Faktor beim Bisulfataufschluß ist, durch das Experiment zu beweisen, wurden drei Versuchsreihen von Probeaufschlüssen hergestellt.

Die erste Reihe besteht aus Aufschlüssen von $100 \mathrm{~g}$ Rohphosphat mit der theoretis $\mathrm{ch}$ erforderlichen Menge Säure verschiedener Konzentration.

Die Aufschlüsse der zweiten Reihe sind mit nur f ü nf $S$ e c h s t el der theoretisch erforderlichen Menge der verschieden konzentrierten Säuren ausgefübrt.

Bei der dritten Reihe kam ein Gemisch von Säure und Bisulfat zur Verwendung, welches aus $5 / 6$ der theorotisch not. wendigen $S$ ä uremenge und der dem fehlenden $1 /$ äquivalenten Menge von Bisulfat bestand.

Vor der Mitteilung der Ergebnisse der Versuchsreihe muß erst noch kurz auf die Ausführung der Versuche eingegangen werden.

Es wurde ein kalkreiches Hardenpont-Phosphat für die Aufschlïsse verwandt, wie es die Industrie im Kriege verarbeiten mußte Die Analyse ergab:

$$
\begin{aligned}
& \text { Feuchtigkeit } 0,92 \% \text {. } \\
& \mathrm{P}_{2} \mathrm{O}_{5} \quad 20,22 \%=44,16 \% \quad \mathrm{Ca}_{3} \mathrm{P}_{2} \mathrm{O}_{8} \\
& \mathrm{Al}_{8} \mathrm{O}_{3} \quad \text { Spuren } \\
& \mathrm{Fe}_{2} \mathrm{O}_{3} \quad \mathrm{I}, 6 \% \\
& \begin{array}{lllll}
\mathrm{CO}_{2} & 14,28 \% & =32,47 \% & \mathrm{CaCO}_{3} &
\end{array}
\end{aligned}
$$

Nach den Gleichungen:

a) $\mathrm{Ca}_{3} \mathrm{P}_{2} \mathrm{O}_{8}+2 \mathrm{H}_{2} \mathrm{SO}_{4}=\mathrm{CaH}_{4} \mathrm{P}_{2} \mathrm{O}_{8}+2 \mathrm{CaSO}_{4}$,

b) $\mathrm{CaCO}_{3}+\mathrm{H}_{2} \mathrm{SO}_{4}=\mathrm{CaSO}_{4}+\mathrm{H}_{2} \mathrm{O}+\mathrm{CO}_{2}$

sind theoretisch zum Aufschluß von 100 Teilen des vorliegenden Phosphates erforderlich:

a) 27,88 Teile $100 \% \mathrm{H}_{2} \mathrm{SO}_{4}=41,8$ 'Teile $\mathrm{H}_{2} \mathrm{SO}_{4}$ von $53^{\circ}$ Ré

b) 31,87 Teile $100 \% \mathrm{H}_{2} \mathrm{SO}_{4}=47,74$ Teile $\mathrm{H}_{2} \mathrm{SO}_{4}$ von $53^{\circ}$ Bé.

Dazu kommt noch der übliche Zuschlag von $5 \%$ Säure für die übrigen im Phosphat anwesenden Basen, so daß theoretisch nötig sind:

$$
41,8+47,74+4,48=94,02 \text { Teile } \mathrm{H}_{2} \mathrm{SO}_{4} \text { von } 53^{\circ} \text { Bé. }
$$

Der Aufschluß von $100 \mathrm{~g}$ Rohphosphat geschah in einer Porzellanschale; der Aufschluß wurde auf dem Trockenschrank bei $60-70^{\circ}$ 12 Stunden lang getrooknet. Wir wissen nun sehr wohl, daß die bei diesem Laboratoriumsauf $s$ chluß angewendeten Säuremengen und die sonstigen Verhältnisse nicht denen beim Aufschluß in der Kammer entsprechen, unsere Aufschlüsse sind aber alle unter genau denselben Bedingungen hergestellt, sie sind reproduzierbar und vergleichbar. Wir sind jetzt dabei, die Verhältnisse des Aufschlusses von Phosphaten genauer zu studieren und werden darüber später berichten. Die in der vorher angegebenen Weise vorgenommenén Aufschlüsse zeigen immerhin deutlich, daß das, was man bei den Bisulfataufschlüssen theoretisch voraussehen konnte, praktisch wirklich eintritt.

Beispiel:

$100 \mathrm{~g}$ Hardenpont-Phosphat In $100 \mathrm{~g}$ des erzeugten Superphos phats sind:

$94 \mathrm{~g}$ Säure $53^{\circ}$ Bé

$194 \mathrm{~g}$ $13,98 \mathrm{~g}$ Gesant- $\mathrm{P}_{2} \mathrm{O}_{5}$

$49,4 \mathrm{~g}=25,47 \%$ Schwund

$144,6 \mathrm{~g}$ Superphosphat
$10,80 \mathrm{~g}$ wasserl. $\mathrm{P}_{2} \mathrm{O}_{5}=77,26 \%$ 1,31 g citratl. $\mathrm{P}_{2} \mathrm{O}_{5}=9,36 \%$ Gesamtaufschl. $86,62 \%$ 
Reihe 1. Theoretische Menge Schwefelsäure.

\begin{tabular}{|c|c|c|c|c|c|c|c|c|}
\hline \multirow{2}{*}{$\begin{array}{l}\text { Konzen- } \\
\text { tration } \\
\text { Grade } \\
\text { Bé. }\end{array}$} & \multicolumn{3}{|c|}{ in $110 \mathrm{~g}$ Superphosphat } & \multirow[b]{2}{*}{$\begin{array}{c}\text { Sehwund } \\
\mathrm{g}\end{array}$} & \multicolumn{3}{|c|}{ Ausbeute } & \multirow{2}{*}{$\begin{array}{l}\text { Unauf- } \\
\text { geschi. } \\
\mathrm{P}_{2} \mathrm{O}_{3} \\
\%_{6}\end{array}$} \\
\hline & $\begin{array}{c}P_{2} \mathbf{O}_{5} \\
\text { Gesaumt } \\
\quad \mathrm{g}\end{array}$ & $\underset{\mathbf{g}}{\mathbf{p}_{\mathbf{z}}^{\mathrm{P}_{2} \mathrm{O}_{\mathbf{o}}}}$ & $\underset{g}{\mathbf{e}_{2} \mathrm{P}_{\mathbf{5}} \mathrm{O}_{\mathbf{5}}}$ & & $\begin{array}{c}\mathrm{P}_{2} \mathrm{O}_{8} \\
\text { wasser!. } \\
\%\end{array}$ & 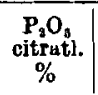 & $\left|\begin{array}{c}\mathbf{P}_{\mathbf{s}} \mathbf{O}_{\mathbf{s}} \\
\mathbf{s u m m}\end{array}\right|$ & \\
\hline 5 & & & & & & & & \\
\hline & & & & & & & & \\
\hline & & & & & & & & \\
\hline \multirow[t]{2}{*}{31,3} & & & & & 68 , & & & \\
\hline & \multicolumn{8}{|c|}{ Reihe 2.} \\
\hline 53 & & & & & 58, & & & \\
\hline & & & & & & & & \\
\hline & & & & & & & & \\
\hline 31,3 & & & & 100 & & 16,68 & & 28,31 \\
\hline \multicolumn{9}{|c|}{ Reihe 3. $5 / 6$ der Schwefe'sälre $+1 / 6$ der Säure als B lsulfat. } \\
\hline 53 & & & & & 66,56 & & & \\
\hline & & & & & & & & \\
\hline & & & & & & & & \\
\hline 31,3 & 11,22 & 8,22 & & 92,48 & 73,25 & 15,35 & 88,60 & 11,4 \\
\hline
\end{tabular}

Aus den vorstehend angegehenen Zahlenreihen läßt sich nun folgendes entnehmen. Der schwund nimmt in allen drei Reihen mit abnehmender Grädigkeit der säure naturgemäß zu. Der bei unseren Laboratoriumsaufsehlüssen in $100 \mathrm{~g}$ Superphosphat gefundene Anteil an citratlöslicher Phosphorsäure ist nur verhältnismäßig geringen Schwankungen mit mehr Schwefelsäure unterworfen; dieser An teil erscheint beim Aufschluß im Großen als wasserlösliche Phosphorsäure. Wir können deshalb wohl ohne weiteres für unsere Betrachtungen die Summe an wasser- und citratlöslicher Phosphorsäure zugrunde legen; in der graphischen Darstellung, Tufel 2, sind demnach als prozentische Ausbeuten diese Summen als Gesantanfschluß eingezeichnet.

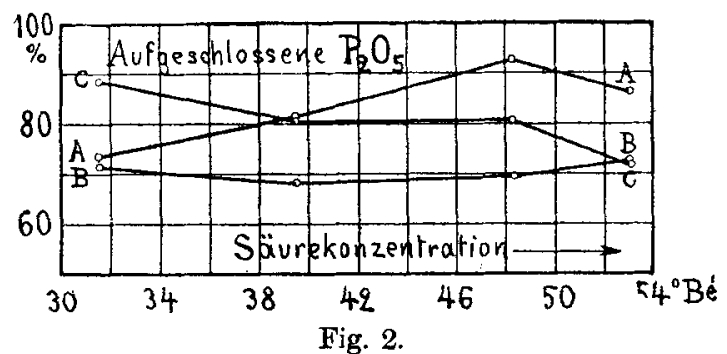

AA. Bei Verwendung der theoretischen Menge reiner Säure verschiedener Konzentration liegt das Maximum des Aufschlusses be einer Säure von etwa $48^{\circ}$ Bé. und nicht etwa bei der höchsten Säurekonzentration.

BB. Bei Verwendung von $5 /$ der thcoretisch erforderlichen Säuremenge ist der Aufschluß im allgemeinen natürlich schlechter, er bleibt bei Säuren bis zu $48^{\circ}$ Bé. annähernd auf gleicher Höhe und wird nur bei stärkeren Säuren um eine Kleinigkeit besser.

CC. Ein ganz anderes Bild ergeben die Aufschlüsse mit dem Ge. misch von Säure and Bisulfat aus $5 / 6$ Säure und $1 / 6$ Bisulfat. Bei ver. dünnten Säuren bis zu $39^{\circ}$ Bé. ist der Aufschluß besser wie bei der theoretischen Säuremenge, bei stärkeren Säuren aber wesentlich schlechter und namentlich über $48^{\circ}$ Bé fällt derselbe besonders ab; bei $521 / 3^{\circ}$ Bé. ist er sogar noch schlechter wie der mit $5 / 6$ säure allein.

Unsere Laboratoriumsaufschlüsse bestätigen also vollständig das, was durch die Löslichkeitsverhältnisse des Bisulfats in Schwefelsäure verschiedener Konzentration festgestellt war.

Beachtenswert ist auch noch der Umstand, daß bei den Aufschlüssen mit der theoretischen Menge reiner Säure der beste Auf schluß nicht bei der höchsten Säurekonzentration von $53^{\circ}$ Bé. liegt, sondern einige Grade darunter. Diese Tatsache beweist, daß die Güte des Aufschlusses nicht nur von der angewandten Menge der Säure, sondern auch von der Konzentration der Säure abhängt, und zwar in der Weise, daß ein bestimmtes. Phosphat für den besten Auf. schluß eine gewisse Menge Säure von ganz bestimmter Konzentration verlangt, die offenbar von der Natur und der Menge der im Phosphat enthaltenen Nebenbestandteile abhängt. Jedes Phosphat will also, wie $S \mathrm{chu} c \mathrm{~h} t$ sich ausdrückt, individuell behandelt sein.

Unsere Untersuchungen ergeben also, da $B$ bei Verwendung von Bisulfat als Schwefel säureersatz die Ausbeuten an aufgeschlosse. ner Phosphorsäure nur eine Besserung or. fahren könnten gegenüber dem AufschluB mit reiner sänre, wenn man mit säurekon zentrationen unter $39^{\circ}$ Bé. arbeiten könnte, bei stärker konzentrierten Säuron fallendie A uf rehlïsse mit steigender Konzentration zunehmend schlechter aus.

Aus unseren Zahlenreihen läbt sich nun auch zahlemmäBig di prozentuelle Wirksamkeit vou Bisulfat als AufschluBmittel im Vergleich mit dem ersetzten Anteil Schwefelsäure für die verschiedeneu Säurekonzentrationen und das vorliegende Hardenpont-Phosphat berechnen. Dabei ergibt sich folgende Gegenüberstellung:

$$
\begin{array}{ccccc}
\text { Grade } \mathrm{Pé.} & 53 & 48,3 & 39,5 & 31,3 \\
\hline \% \mathrm{H}_{2} \mathrm{SO}_{4} & -3 & +50,71 & +97,55 & +1150
\end{array}
$$

Die säure von $53^{\circ} \mathrm{Bé}$, bei welcher $1 / 6$ durch Bisulfat ersetzt ist, schlielst also um noch $3 \%$ schlechter auf als die gleich konzentrierte Säure, wenn nur $\%$ der theoretischen Menge zur Anwendung kommen. Bei einer Säure von $48^{\circ}$ Bé. vermag das Bisulfat etwa die Hälfte des fehlenden $1 / 6$ Säure zu ersetzen, bei $39^{\circ}$ Bé. schließt die mit. Bisulfat gestreckte Säure fast ebenso gut auf wie die nicht gestreckte, und erst bei dünneren Säuren wächst die Wirksamkeit des Bisulfats; sie er-

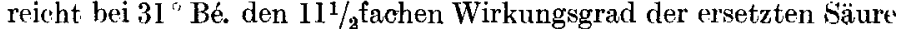

Bei der Superphosphatfabrikation sind hauptsächlich zwei Be. dingungen zu erfüllen; nämlich eine möglichst vollkommene Überführung der im Rohphosphat enthaltenen Phosphorsäure in Monocalciumphosphat, und die Erzeugung eines gut streufähigen Produk tes. Wie das Bisulfat in bezug auf den Aufschluß wirkt, ist oben gezeigt worden, wie es die physikalische Beschaffenheit des Superphosphats beeinflußt, soll nachstehend untersucht werden.

Beim Aufschluß von Phosphaten bildet sich Gips, dieser bindet in der Kammer ab und bildet gewissermaßen das Skelett, in welchem das Monocalciumsalz cingebertet jst. Der Gips verhindert also das Verschmieren der Ware bei der Zerkleinerung, das Zusammenbacken in der Verpackung, er bedingt also in der Hauptsache die Streufähig. keit.

Bei unseren Laboratoriumsaufschlüssen wurde, wie angegeben, absichtlich eine mehrstündige Trocknung ausgeführt, um immer ein trockenes, für die Analyse geeignetes Material zu haben. Dadurch wurde allerdings die Möglichkeit einer Beurteilung der Festigkeit des Aufschlußproduktes stark vermindert. Es ließ sich nur feststellen, daß die mit Bisulfatzusatz erhaltenen Aufschlüsse einen größeren Feuchtigkeitsgehalt aufwiesen, als die mit reiner Säure hergestellten.

Bei Säurekonzentrationen bis zu $45^{\circ}$ Bé. herab ging die Reaktion auch bei gewöhnlicher Temperatur sehr lebhaft vor sich, bei den niedrigeren Konzentrationen blieb sie sehr matt, und erst bei Wärmezufuhr begann eine nennenswerte Einwirkung. Aufschlüsse mit dünnen Säuren zeigten Neigung zur Klumpenbildung. Bisulfatzusatz wirkte stark verzögernd auf die Reaktion. Im ersten Augenblicke trat zwar eine ganz kurze, lebhafte Einwirkung auf, die aber sofort abflaute und schlecht blieb. Erst bei längerer Erwärmung setzte die Reaktion allmählich cin, blieb aber auch da bis zum Ende recht mäßig.

Diese Beobachtungen können also nur sehr unvollkommen AufschluB auf die Frage geben, wic das Bisulfat die Skelettbildung beeinflußt. Wir haben deshalb einen anderen Weg eingeschlagen.

Es wurde versucht, die Abbindeverhältnisse von Gips, allein, und mit Zusatz verschiedener Mengen von Bisulfat, mit einem $V$ i c a $t$. sehen Nadelapparat, wie er in der Zementindustrie verwendet wird, festzustellen, d. h. es wurde die Zeit gemessen, innerhalb welcher eine bestinmte Menge Alabastergips (154 g Gips auf $100 \mathrm{ccm}$ Wasser) abbindet und wie die Abbindezeit sich ändert, wenn dieser Gipsmenge, in Abständen von $5 \%$ steigend Zusätze von Bisulfat gemacht werden. Als Abbindezeit galt dabei das Zeitintervall von dem Augenblick ab, wo die Nadel gerade nicht mehr den Boden des Apparates er. reichte, bis zu dem Moment, wo sie nicht mehr in die Oberfläche ein. dringt. Die Messungen ergaben folgendes:

\begin{tabular}{c|c|c|c|c|c}
\hline & $\begin{array}{c}\text { ohne Bi- } \\
\text { sulfat }\end{array}$ & $\begin{array}{c}5 \% \mathrm{Bi}- \\
\text { sulfat }\end{array}$ & $\begin{array}{c}10 \% \mathrm{Bi}- \\
\text { sulfat }\end{array}$ & $\begin{array}{c}15 \% \mathrm{Bi}- \\
\text { sulfat }\end{array}$ & $\begin{array}{c}20 \% \mathrm{Bi}- \\
\text { sulfat }\end{array}$ \\
\hline $\begin{array}{c}\text { Abbindezeit } \\
\text { in Min. }\end{array}$ & 6 & 1,5 & 2 & 3,5 & $\begin{array}{c}\text { bindet un- } \\
\text { vollst. ab }\end{array}$
\end{tabular}

Diese Verhältnisse sind in der umstehenden Tafel 3 graphisch dargestellt.

Man erkennt, daß der reine Gips 6 Min. zum Abbinden braucht, daß dagegen bei $5 \%$ Zusatz ron Bisulfat die Abbindezeit auf 1,5 Min. zurückgeht und bei weiteren Zusätzen bis zu $15 \%$ wieder langsam steigt. Bei 20\% Zusatz bindet der Gips nur noch teilweise ab. 
Daß Gips bei Zusatz von wenigen Prozenten eines gut dissoziierbart $n$ Salzes viel schneller abbindet als in reinem Zustande, ist bekannt, and die Praxis macht davon Gebrauch. Diesen Vorgang hat R o h I a $\mathrm{ll}^{\left.\mathbf{a}^{3}\right)}$ zu erklären versucht. In unserem Falle wird bei der Reaktion zwischen Rohphosphat, Schwefelsäure und Bisulfat $\mathrm{Na}_{2} \mathrm{SO}_{4}$ frei. Die leichte Krystallisierbarkeit dieses Salzes beschleunigt offenbar dus Abbinden des Gipses; geht man aber mit dem Zusatz über 5\% hinauk, so wirkt das Salz in stejgendem Maße nur noch als Verdiunnungsmittel, und schließlich wird der Gips so stark damit durchsetzt, daß er nicht mehr abbindet. Die Abbindeproben nehmen rein äußer.

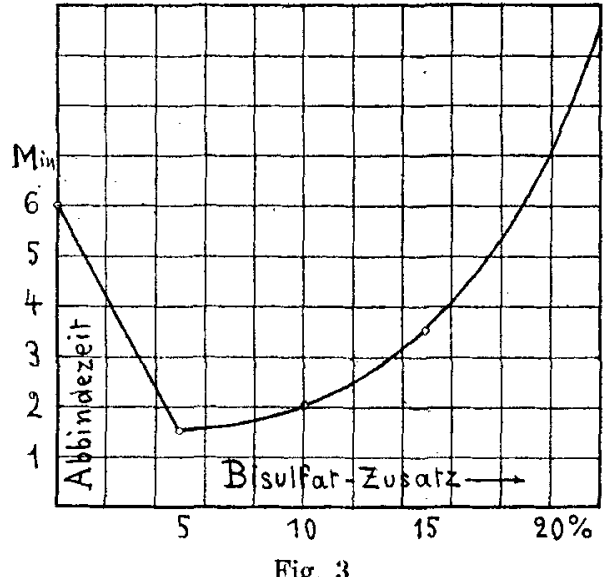

Fig. 3.

lich mit steigendem Bisulfatzusatz mehr und mehr an Porosität zu und lassen sich durch geringen Druck zu schmierigen Massen formen.

Bei dem vorliegenden Hardenpont-Phosphat mit seinem hohen $\mathrm{CaCO}_{3}$-Gehalt sind die Bedingungen für die Gipsbildung noch recht günstige, aber auch hier ist bereits bei Verwendung von $1 / 6$ Bisulfat das Verhältnis von Bisulfat zum entstehenden Gips wie 19,66: 100. Es ist also bereits die Grenze erreicht, bei welcher die Beschaffenheit des Gipsskelettes und damit die Streufähigkeit des Superphosphat ungünstig beeinflußt wird. Bei carbonatairmeren, besseren Rohphosphaten würde die Erscheinung noch wesentlich mehr ins Gewicht fallen.

$$
\text { Zus a m m en assung. }
$$

Durch Löslichkeitsbestimmungen von Bisulfat in Sohwefelsäure verschiedener Konzentration wird gezeigt, duB die säurekonzentration durch diesen Zusatz nur bis zu einer Konzentration von $47,5^{\circ}$ Bé zunimmt, darüber hinaus sich sogar verringert. Bisulfat käme prak tisch als Ersatz für Schwefelsäure nur in Frage, wenn man mit niedrigen Säurekonzentrationen von etwa $30^{\circ}$ Bé. an abwärts arbeiten könnte.

Diese theoretisch vorauszusehenden Verhältnisse werden durch vine Reihe von Laboratoriumsaifschlüssen bestätigt.

Weiter wird noch der Einfluß des Bisulfats auf das Gipsskelett und die Streufähigkeit des Superphosphats untersucht und zahlen. näßig festgestellt.

[A. 197.]

\section{Zur Geschichte des synthetischen Kautschuks.} Von Professor Dr. Fritz Howminn, Breslau.

$$
\text { (Eingeg. 24./1. 1؟20.) }
$$

Währerd des Weltkrieges haben dio Farbenfabriken rorm Friedrich Bayer \& Co., Elberfeld-Leverkusen erhebliche Mengen Methylkautschuk hergestellt ${ }^{1}$ ). Damit hat die Kautschuksynthese ihre technische Feuerprobe bestanden. Wie zahlreiche dentsche und österreich-ungarische Gummiwarenfabriken sich mit diesem künstlichen Material praktisch auseinandergesetzt haben, zeigte in ausführlicher Weise $K u r t$ Gott $l o b^{2}$ ). Nach dem deutschen Zusammenbruch ist auch die Fabrikation dieses Kriegsgummis zum Erliegen gekommen. Doch sehe ich in der Hoffnung, daß der Wellenzug der Entwicklung auch aus diesem Tale wieder auf den Berg führen wird, wo Weiterarbeit an dem alten Probleme ge. boten ist, mehr als weltfremden Optimisnus. Der Stand unserer Valuta sorgt ja dafür, daß der britische Plantagenkautschuk bei uns in Deutschland noch immer nicht. für den Märchenpreis von

3) Z. anorg. Chem. 31, 347 [1902]; 35, 199 [1903]; 36, 335 [1903].

1) C. D u is be r.g, Gummi-Ztg. 33, 158 [1918].

2) K. Gott $1 \circ$ b, Gumni-Ztg. 508-590. 524-535, 551-553. $576-577,599-600$.
$2 \mathrm{M}$ je Kilo zu handeln ist. Je früher hier wieder die praktische Tätigkeit einsetzt, je besser ist es für uns und unsere notleidende Volkswirtschaft.

Über den Werdegang der synthetischen Kautschuke ist vor der Hauptversammlung des Vereins deutscher Chemiker in Freiburg im Mai 1912 eingehend berichtet worden ${ }^{3}$ ). Das Werturteil, dem damals der Vorsitzende des Vereins Ausdruck verlieh ${ }^{4}$, schicn jahrelang allseitige Anerkennung zu finden, denn einen leicht. fertigen Einwand Perkins jum., als ob die Farbenfabriken von dem Engländer $H$ e i n e w a n n ihre Patentansprüche entlehnt hätten, konnte ich glatt.durch Beweis aus den Patentakten ad absurdum führen ${ }^{5}$ ). Erst in jüngster Zeit hat sich eine Stimmo gegen das bisher Anerkannte erhoben. Da Carl Harries os ist, der sich vernehmen läßt, einer der angesehensten Kautschukforscher Deutschlands, kommt dieser Äußerung natürlich ein besonderes Gewicht zu. Er wird gute Gründe beibringen, die ihn veranlassen, von dem bisher für richtig Gehaltenen abzuweichen. Hören wir seine Ausführungen:

In seinen bei Julius Springer 1919 erschienenen ,Untersuchungen über die natürlichen und künstlichen Kautschukarten " behandelt $\mathrm{H}$ a $\mathrm{r} \mathrm{r}$ ies in der II. Abteilung, Kapitel I, Seite $125 \mathrm{ff}$.: „Die ge schichtliche Entwicklung der künstlichen Darstellung der Kaut. schuke und ihrer Verwandten". Auf Grund einer Äußerung C. D u i s . b ergs vor der Deutschen Bunsengesellschaft nimmt $H$ a $r$ ies nun die Priorität der Wärmepolymerisation, die mir bisher zuge. billigt wurde, für sich in Anspruch. Der fragliche Satz D u i s bergs lautet in Original: ,Die synthetische Darstellung des Kautschuks ist vielfach versucht worden und wurde in Leverkusen in den Jahren 1910 bis 1912 von Fritz $\mathbf{H}$ of $\mathrm{fm}$ a $\mathrm{n} \mathbf{n}$, fußend auf den Untersuchungen $\mathrm{H}$ ar $\mathrm{r}$ ies, ausgeführt ${ }^{\text {s/6 }}$ ). Wenn $\mathrm{D} u$ is . berg in dieser Sache nichts weiter gesprochen hätte, als diesen einzigen Satz, so wäre die von $H$ ar 1 i es gezogene Folgerung: „Hierin sehe ich jetzt meine Priorität in bezug auf die Wärmepoly. merisation anerkannt", vielleicht zu verstehen; denn wenn ein Schüler auf den Untersuchungen seines Meisters fußt, so ist dieser letzten Endes der Gebende, und ihm gebührt der Preis. Die Elber. felder Kautschukarbeiten haben sich aber ganz anders abgespielt, und $D \mathrm{u}$ is be $\mathrm{r} g$ selber hat wiederholt eingehend über den Ver. lauf dieser Dinge gesprochen und hat immer klar zum Ausdruck gebracht, wie die Rollen verteilt waren, was $\mathrm{H}$ arries gebührte, und was den Chemikern der Farbenfabriken zukam. Wir haben ganz und gar nicht auf $\mathrm{H}$ ar r i es' Arbeiten gefußt. Ich persönlich trat sogar von Anfang an in bewußten Gegensatz zu seinem Axiom vom Dimethylcyclooctadienring, da ich für diese Hypothese nirgends eine Begründung sah. Nur wenn wir uns diesen Achterring. gedanken zu eigen gemacht hätten, wenn wir seiner Verwirklichung nachgestrebt, könnte von einer Gefolgschaft $\mathrm{H}$ a r r i e s' gesprochen werden. Mündlicher oder schriftlicher Gedankenaustausch hat mit Harri es bis 1909 nicht stattgefunden. Über natürlichen Kautschuk, dessen Studium H a r ries viel beschäftigt hat, arbeiteten wir nicht, für die Ozonarbeiten fehlte uns die Apparatur. Auf welchen Arbeiten hätten wir dann fußen sollen? Ich weiß keine zu nennen. Wenn $D$ u is berg trotzdem vor der Bunsengesellschaft seine oben zitierte Wendung gebrauchte, so wollte er damit meines $\mathrm{Er}$. achtens die unbestreitbaren Pionierverdienste des in der Sitzung anwesenden $\mathrm{H}$ a r ries würdigen, nicht aber zum Ausdruck brin gen, daß seine Elberfelder Chemiker nur Kopisten früherer H a r r i s scher Arbeiten waren. Wir sind so weit selbständig auf diesem Gebiete vorgeschritten, wie es bei einem Problem mög lich war, um das sich schon ganze Chemikergenerationen bemüht haben. (Ich verweise hier nur auf die vortrefflichen und das Thema erschöpfenden Ausführungen von $\mathbf{P}$ o n d.) 'Ich glaube, daß damit die Bedeutung des $\mathrm{D}$ u is bergschen Satzes auf das richtige Maß zurückgeführt ist, daß er, so interpretiert, den Sinn hat, den der Redner ihm untergelegt. Aber $\mathbf{H}$ a r r i e s führt weitere Gründe für seine Prioritätsansprüche ins Feld. Er erbliokt sie in Arbeiten die er bereits 1902 veröffentlicht hat ${ }^{7}$ ). Sic betreffen die Polymeri-

3) Carl Harries, Angew. Chem. $25,1457-1462$ [1912]; u. Fritz H of ma $n$, Angew. Chem. 25, J462-1467 [1912]

4) Angew. Chem. 25, 1858 [1912].

5) Angew. Chem. 25, 1858 [1912].

b) Einige kleine Irrtümer in diesem Satz lassen die Vermutung $\mathrm{zu}, \mathrm{da} B$ es sich um eine Improvisation des Redners gehandelt hat denn nicht Leverkusen, sondern Elberfeld, nicht 1910-1912, sondern Sommer 1909 und nicht $\mathrm{H}$ of $\mathrm{f} \mathrm{m}$ a $\mathrm{n} \mathrm{n}$, sondern $\mathrm{H}$ of $\mathrm{m}$ a $\mathrm{n} \mathrm{n}$ soll es heißen.

7) C. H a r r i s s. M. W e iss, Ber. 35, 3265 [1902]. 